\title{
Un acercamiento a la situación de la salud bucal en Uruguay
}

\section{An approach to the oral health situation in Uruguay}

\section{Nota Científica}

Sylvia Piovesan Suárez ${ }^{1, a, b}$

${ }^{1}$ Universidad de la República, Facultad de Odontología, Montevideo, Uruguay.

a Magister en Enseñanza Superior, Magister en Bioética. b Investigadora. Presidenta SUIO (Sociedad Uruguaya de Investigación Odontológica).

\section{Correspondencia:}

Sylvia Piovesan Suárez: sylvia.piovesan@gmail.com General las Heras 1925, 11600 Montevideo, Departamento de Montevideo, Uruguay

ORCID: 0000-0003-1807-6380

\section{Editora invitada:}

Lita Ortiz-Fernández

Universidad Nacional Mayor de San Marcos, Perú.

Conflicto de intereses: la autora declara no tener conflictos de interés.

Fuente de financiamiento: autofinanciado.

Recibido: $27 / 04 / 20$

Aceptado: $11 / 05 / 20$

Publicado: 04/08/20

\begin{abstract}
Resumen
El estado de salud bucal en Uruguay fue conocido luego del relevamiento nacional poblacional realizado entre 2010 y 2011. Las patologías más prevalentes detectadas fueron la caries y las paradenciopatias. Además, se estudió maloclusiones, pérdida dentaria y lesiones de la mucosa. La información se complementó con otros estudios epidemiológicos, como el realizado en relación con trastornos témporo mandibulares, que evidenció que la mitad de la población uruguaya presenta síntomas y/o signos vinculados con dicho desorden. El Sistema Nacional Integrado de Salud, que funciona desde 2008, concentra efectores públicos y privados y ha intentado cumplir con un Programa Nacional de Salud Bucal. Brinda prevención, promoción y tratamiento de patologías orales de complejidad menor, por lo que quedan muchas prestaciones por fuera del sistema. La formación de recursos humanos es suficiente para cubrir las necesidades de la población, pero la odontología en general, y la especializada aún más, resultan muy costosas y no son accesibles a todos los que la precisan.
\end{abstract}

Palabras clave: Salud bucal; Salud pública; Odontología preventiva; Uruguay (fuente: DeCS BIREME).

\section{Abstract}

Oral health status in Uruguay was known after the national population survey carried out between 2010 and 2011. The most detected prevalent pathologies were caries and paradenciopathies. In addition, malocclusions, dental loss and mucosal lesions were studied. The information was complemented with other epidemiological studies, in relation with to temporomandibular disorders, which showed that half of the Uruguayan population presents symptoms and / or signs related to said disorder. The National Integrated Health System, which has been operating since 2008, concentrates public and private effectors and has attempted to comply with a National Oral Health Program. It provides prevention, promotion and treatment of oral pathologies of lesser complexity, so many benefits remain outside the system. Training human resources is sufficient to meet the needs of
\end{abstract}


the population, but dentistry in general, and specialized, are very expensive and are not accessible to all who need it.

Keywords: Oral health; Public health; Preventive dentistry; Uruguay (source: MeSH NLM).

\section{Introducción}

Uruguay es el país de Sudamérica situado a la margen izquierda del río Uruguay, de donde deriva su nombre completo, República Oriental del Uruguay. Está ubicado también a la margen izquierda del río de la Plata hasta su desembocadura en las aguas oceánicas del Atlántico que bordean el tramo más este de la costa sur uruguaya.

Estos dos ríos trazan el límite con la República Argentina por el lado oeste y suroeste, mientras que por el norte y el este, Uruguay hace límite con la República Federativa del Brasil. A diferencia de la anterior, esta frontera es seca en su mayoría, por lo que varias poblaciones de ambos países comparten un espacio territorial y cultural propio (Figura 1).

La superficie terrestre es de aproximadamente 175016 kilómetros cuadrados y en 2018 la población era de 3 505985 habitantes, de los cuales 1381946 viven en la capital del país, Montevideo. Estos datos pertenecen al informe del año 2019 del Instituto Nacional de Estadística (INE) ${ }^{1}$.

La mencionada distribución de la población ha condicionado una presencia de recursos sanitarios, tanto materiales como humanos, mayoritariamente concentrados en Montevideo.
En búsqueda de un sistema de salud más equitativo, integral y accesible, Uruguay reformó su sistema sanitario en 2008 creando el Sistema Nacional Integrado de Salud (SNIS), y a partir de entonces, se articularon los sistemas público y privado para asistir a toda la población ${ }^{2}$.

En esta nueva estructura sanitaria, la salud bucal, entendida como parte de la salud general, fue incluida dentro del Programa Nacional de Salud Bucal. Este programa priorizó a embarazadas y menores de edad, fundamentalmente ${ }^{3}$.

La atención odontológica comprendida en el SNIS hace cambiar, aunque parcialmente, la forma de la asistencia odontológica predominante en Uruguay, la cual se realizó durante el siglo pasado, de forma casi exclusiva, en el ámbito privado (consultorio particular) y en las últimas décadas, incorporando modalidades colectivizadas pero de carácter privado, en su mayoría. Salud pública y las instituciones médicas privadas cubrían urgencias odontológicas y cirugía.

En concordancia con estos cambios de estructuración de la atención de salud por el SNIS, la academia y, en particular, la Facultad de Odontología de la Universidad de la República (FO.UdelaR) responsable de la formación de la mayoría de los recursos humanos en Odontología, llevó adelante una importante investigación. El proyecto

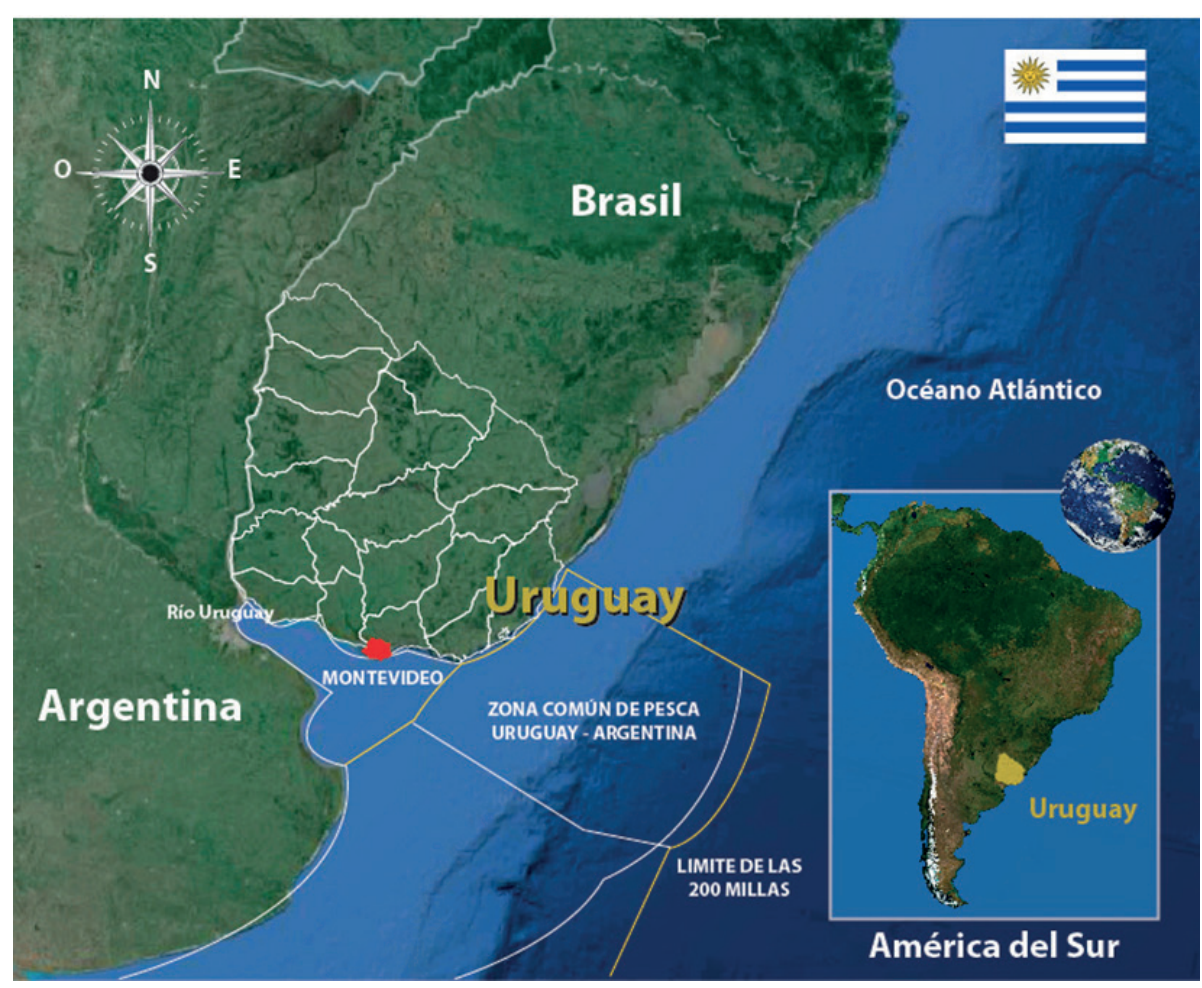

Figura 1. Ubicación de la República Oriental del Uruguay (ROU)

Fuente: Anuario 2019 del INE 
fue realizar el primer relevamiento de las enfermedades orales más prevalentes en la población uruguaya, el cual efectivamente se ejecutó entre 2010 y $2011^{4}$. En ese mismo sentido, se hizo otro estudio epidemiológico poblacional para conocer la prevalencia de trastornos témporo mandibulares (TTM), en todo el país, que mostró cuán prevalente es esta patología en Uruguay ${ }^{5}$. Otras indagatorias sobre grupos particulares, caries en niños, población en tratamiento por drogodependencia, niños diabéticos, etc. ${ }^{6-8}$, aportaron conocimiento del estado de salud oral de diferentes colectivos uruguayos en los últimos diez años.

El presente artículo intenta dar información con sentido y fundamento ético - social de la situación de la salud bucal en Uruguay, y ser un aporte a la comunidad odontológica latinoamericana para la mejora del bienestar de sus poblaciones.

Se expone como un tema circular en el sentido que, primero, presenta cómo se planteó la búsqueda del conocimiento desde la academia para retornar al punto de partida y volcar a la sociedad un tema de interés general; saber cuál era el estado real de la salud bucal de la gente fue tarea propiciada por la Facultad de Odontología de la Universidad de la República. Desde una segunda perspectiva, sirvió para dar luz a quienes tienen el poder y deber de instalar y sustentar políticas sanitarias. Tercero, y no menos importante, fue utilizada para planificar la formación de odontólogos, higienistas, asistentes dentales y laboratoristas con la actualización de planes de estudio y un perfil profesional adecuado a la realidad. Los egresados podrían insertarse en un mundo laboral, sin olvidar que sus profesiones han sido siempre de servicio y podrían adaptarse a las necesidades bio-sociales, económicas y culturales concretas de las personas para ejercer su profesión.

\section{Estado de la salud bucal en el país: prevalencia y seve- ridad de las principales enfermedades bucales}

La academia. En Uruguay existen solamente dos instituciones en la formación de grado y postgrado en Odontología y auxiliares de Odontología: en el ámbito público, la Facultad de Odontología de la Universidad de la República (FO.UdelaR) fundada en 1929, y en el sector privado, la Facultad de Odontología de la Universidad Católica del Uruguay Dámaso Antonio Larrañaga (UCUDAL) que fue inaugurada en el año 2000. La trayectoria acumulada en más años de formación de profesionales, la capacidad edilicia y, sobre todo, el factor económico, hace que la mayoría de los odontólogos se formen en la Facultad pública.

En este apartado, la presentación de las dos casas de estudio se realiza para ubicar al lector en dónde se concentra el conocimiento de odontología en el país. Existen también dos agrupaciones profesionales: la Asociación Odontológica Uruguaya (AOU), que asocia odontólogos egresados de ambas facultades, y la Federación Odontológica del Interior (FODI) que agrupa odontólogos que ejercen fuera de Montevideo. Además, la Sociedad Uruguaya de Investigación Odontológica
(SUIO) División de la International Association for Dental Research $(I A D R)$, la cual es una asociación civil para la investigación odontológica, contribuye en la formación, actualización e investigación odontológica, junto a las anteriores.

Luego de la breve mirada del ambiente odontológico nacional, se explica cómo fue que se pudo conocer científicamente cuál era el estado de salud bucal de los uruguayos, a partir de las instituciones.

El manual de encuestas básicas de la Organización Mundial de la Salud (OMS) ${ }^{9}$ expresa la importancia de contar con estudios epidemiológicos poblacionales periódicos para planificar y evaluar programas de salud bucodental, impulsando a las autoridades sanitarias a que lo realicen y, en su defecto, que sean las instituciones académicas quienes lo lleven a cabo.

En ese sentido, Uruguay realizó el estudio que cumple dichas características gracias a la iniciativa de la FO.UdelaR que tuvo la responsabilidad de dirigir el relevamiento nacional y que contó con el auspicio del Ministerio de Salud Pública (MSP) en el período 2010-2011.

Este proyecto se enmarcó dentro de un "Macro Proyecto Institucional" para el desarrollo de la investigación epidemiológica y fue sustentado económicamente, en un $90 \%$, por la Comisión Sectorial de la Universidad de la República, por la Sociedad Uruguaya de Investigación Odontológica (SUIO), en un 7\%, y por Colgate Palmolive-Uruguay, en un 3\%. Se destaca entonces que si bien la autoridad sanitaria MSP dio su auspicio y apoyó la iniciativa, no invirtió recursos financieros para el proyecto.

Hallazgos del primer relevamiento nacional de jóvenes y adultos uruguayos. El estudio de carácter transversal y descriptivo se realizó con un muestreo conglomerado estratificado en todo el territorio uruguayo registrándose variables no solamente odontológicas, sino también sociodemográficas y conductuales.

Para caries se utilizó el índice CPO, cariados, perdidos, obturados con el criterio de la OMS de lesión cavitada; para paradenciopatías, fue el Índice Periodontal Comunitario (CPI); para maloclusiones el Índice Estético Dental (DAI). El relevamiento también incluyó lesiones de la mucosa con especial atención en las lesiones premalignas y malignas.

La caries resultó el principal problema de salud oral en los jóvenes de 15 a 24 años. Las paradenciopatías prevalecieron en los adultos entre 35 a 44 ańos, y la pérdida dentaria, en los adultos mayores de 65 años ${ }^{4,9-13}$.

A continuación se presenta en forma resumida los hallazgos sobre caries de acuerdo a la investigación de Lorenzo et al ${ }^{4,9}$.

Prevalencia de caries. El 81\% de los jóvenes tienen experiencia de caries, mientras que, en los adultos, es el $100 \%$. Con respecto a la región geográfica, se encontró que la prevalencia es menor en Montevideo que en el resto del país ${ }^{4}$. La severidad de la caries medida con el 
índice CPOD de acuerdo al grupo etario se presenta en la Tabla 1.

Prevalencia de paradenciopatías. En cuanto a la presencia de bolsa periodontal (con profundidad de surco gingival > a $4 \mathrm{~mm}$ ), Lorenzo et al. observaron que la prevalencia no supera el $30 \%$ para las edades adultas para ambos tramos etarios ( 35 a 44 y mayores de 65 años) y que ese valor se debe al aumento de pérdida dentaria en los adultos mayores. También, observaron la tendencia de los hombres adultos a tener mayor profundidad de sondaje de bolsa periodontal. Con respecto a los jóvenes, se vio una prevalencia de bolsa significativamente mayor para la región del interior del país ${ }^{4,10}$.

Pérdida dentaria. En relación con la proporción de personas edéntulas, se encontró que la pérdida dentaria en los adultos mayores fue de $27 \%$ y en los adultos de 35 a 44 años, de 1\%. En el tramo etario de jóvenes fue del $0 \%{ }^{4,12}$.

Lesiones de la mucosa. Respecto a las lesiones de la mucosa, se observó un total de $0,09 \%$, con mayor porcentaje en la población del interior, una mayor proporción en los adultos y adultos mayores $(0,12 \%$ y $0,14 \%$ respectivamente) y una menor proporción en los jóvenes $(0,05 \%)^{4,13}$.

Maloclusiones. El 65\% de los jóvenes uruguayos entre 15 y 24 años de edad presentan un nivel leve de maloclusión. Según el índice de estética dental, el promedio de DAI es de 24,49 (95\% IC: 23,88\% - 25,11\%). Es similar en ambos sexos tanto para el interior como en Montevideo ${ }^{4,11}$.

Hallazgos de prevalencia en trastornos témporo mandibulares (TTM). Riva et al. realizaron un estudio descriptivo transversal poblacional con personas de 6 a 70 años. El estudio demostró una alta prevalencia en Uruguay de trastornos témporo mandibulares (TTM) Los porcentajes encontrados en Montevideo y el resto del país superan el 50\%. Estas patologías involucran una serie de variables que impactan sobre la salud del sistema estomatognático y la calidad de vida de los individuos que las padecen. Por ello, los autores estiman que debería considerárselo un problema de salud pública, ya que provoca consecuencias severas en la población que lo sufre 5 .

\section{Otros hallazgos}

Estudios en niños. En 2003, Lorenzo había analizado la prevalencia de caries en niños de 12 años de escuelas públicas de diferentes barrios de Montevideo, y encontró que la descripción del fenómeno caries dental y su vinculación con el nivel socioeconómico de los niños estudiados, coincidía con hallazgos previos. Esto permitió elaborar la hipótesis de la tendencia descendente del fenómeno para Uruguay, pero, a su vez, dio indicios sobre las diferencias entre los diversos grupos de niños, según su nivel socioeconómico ${ }^{14}$.

Posteriormente, Angulo et al. en 2010 estudiaron caries dental, fluorosis y gingivitis en adolescentes escolarizados de 12 años de edad y encontraron que, en Montevideo, la población de niños de 12 años libres de caries era de $55,8 \%$ y en el interior del país, $47,1 \%$, empeorando la situación con un $38,1 \%$ hacia la zona de frontera seca con Brasil ${ }^{6}$.

Lopez Jordi et al. en 2014 estudiaron la hipomineralización en molares de niños ${ }^{15}$.

Álvarez et al. estudiaron la erosión dentaria en $2015^{16}$ a lo que posteriormente en 2017 Caviglia prosiguió en su tesis de maestría al estudiar la misma problemática en niños de 5 años. Encontró que la prevalencia de erosión dentaria en los preescolares uruguayos es alta, 57,6\%, siendo palatino de los incisivos superiores la superficie más afectada. Fue encontrada una asociación significativa con el nivel socioeconómico alto ${ }^{17}$.

Techera et al. realizaron un estudio en niños diabéticos tipo I. Encontraron que existen diferencias estadísticamente significativas en el índice de sangrado entre diabéticos y no diabéticos, pero no en las variables relativas a caries. Este estudio fue de caso-control, observacional y analítico ${ }^{7}$.

Se destaca que en la Cátedra de Odontopediatría de la FO.UdelaR se está desarrollando el proyecto "Prevalencia, extensión y severidad de la caries dental a la edad de 2, 5 y 12 ańos, indicadores de riesgo e impacto en la calidad de vida”, estudio transversal de Uruguay para el Primer Observatorio de Investigación de Caries Dental en América Latina ${ }^{18}$.

Jóvenes en tratamiento por consumo de drogas. En 2014, Rotemberg et al. realizaron estudios en población adolescente y adulta joven entre 15 y 35 ańos en tratamiento por drogodependencia ${ }^{8}$.

Se pudo detectar la prevalencia de patología dentaria y periodontal en el que la media del índice CPOD fue de 8,04 . Al discriminar por franja etaria, la comprendida entre 15 y 24 años tuvo un CPOD de 5,31, mientras que la comprendida entre 25 y 35 años tuvo un valor de 11,27 . El relevamiento paradencial mostró que el $65 \%$ de los participantes presentaron gingivitis y el $18 \%$, cuadros de periodontitis.

Tabla 1. Severidad de caries en Uruguay por grupo etario

\begin{tabular}{lccc}
\hline Grupo etario & CPOD & Femenino & Masculino \\
\hline 15 a 24 años & 4,15 & 4,49 & 3,82 \\
35 a 44 años & 15,2 & 16,47 & 13,84 \\
Mayores de 65 años & 24,13 & 25,27 & 23 \\
\hline
\end{tabular}


Los resultados obtenidos mostraron que existe una mayor prevalencia de enfermedad oral en pacientes drogodependientes que la población general ${ }^{8}$.

Personas afrodescendientes uruguayas. Rotemberg et al. en 2017 estudiaron caries en una población afrodescendiente y la compararon con igual cantidad de individuos no afrodescendientes de Montevideo, y encontraron que la cantidad de dientes perdidos es superior en el grupo afrodescendiente ${ }^{19}$.

Sistema Nacional Integrado de Salud (SNIS). Es importante, y como deber ético de toda investigación epidemiológica en salud, proyectar sus resultados para contribuir a la mejora y atender las condiciones desfavorables sanitarias de una población.

Las investigaciones permiten ver las situaciones reales sobre las cuales adquiere relevancia la toma de decisiones políticas sanitarias. Estas deben encarar las estrategias posibles para prevenir, promocionar la salud y el cuidado, atender las necesidades, mantener el control, evaluar las acciones.

En el caso de Uruguay, a partir de 2008 se creó el nuevo sistema de salud llamado Sistema Nacional Integrado de Salud con la ley 18211. Así, se intenta dar cobertura integral y equitativa a toda la población. En su artículo 1, expresa: "La presente ley reglamenta el derecho a la protección de la salud que tienen todos los habitantes residentes en el país y establece las modalidades para su acceso a servicios integrales de salud. Sus disposiciones son de orden público e interés social" ${ }^{2}$.

Atención en odontología. El SNIS deja en la órbita del MSP, según artículo 5 de la citada ley 18211 inciso E) "Aprobar los programas de prestaciones integrales de salud que deberán brindar a sus usuarios los prestadores públicos y privados que integren el Sistema Nacional Integrado de Salud, y mantenerlos actualizados de conformidad con los avances científicos y la realidad epidemiológica de la población" ${ }^{2}$.

Desde el MSP se establece el Programa Nacional de Salud Bucal cuyos objetivos y alguna de sus aspiraciones se transcriben de la web del MSP.

\section{Objetivos:}

- Contribuir al logro del más alto grado posible de salud bucal de la población uruguaya, impulsando, promoviendo y articulando las adecuadas acciones promocionales, preventivas y asistenciales integradas.

\section{Descripción:}

- Integrar el componente salud bucal en el SNIS. Definir la normativa correspondiente: objetivos sanitarios, definición de prestaciones obligatorias a incluir en el PIAS para todas las Instituciones del SNIS, metas asistenciales, promover la articulación de los prestadores a efectos de cumplir con la nueva normativa, establecer mecanismos de control, formular e implementar Programas Pilotos, que ejecuten la integración del componente salud bucal en el SNIS, contribuir a las transformaciones necesarias del sector servicios de salud bucal en el marco de los objetivos sanitarios del SNIS.

- Definir la política nacional de salud bucal, en términos de normas y orientaciones que incorporen actividades de promoción y asistencia de calidad con criterio poblacional.

- Definir las actividades asistenciales integrales específicas dirigidas a grupos de población: programas dirigidos a gestantes, niñas y niños, adolescentes, adultos/ adultas, y adultos/adultas mayores.

- Definir las actividades asistenciales integrales vinculadas a colectivos: Programas de Salud Colectiva,

- Normatizar el tratamiento de las siguientes situaciones o condiciones de salud enfermedad: caries, paradenciopatías, maloclusiones, patología tumoral y no tumoral de tejidos blandos y duros, VIH-SIDA, enfermedades sistémicas y salud bucal, mutilados dentales por secuelas de caries y paradenciopatías, mutilados maxilo-faciales, discapacitados.

- Definir las lineas estratégicas vinculadas a la promoción y prevención de la salud bucal a nivel nacional. Articulación con los programas nacionales de salud. Definición de una estrategia nacional de comunicación y publicidad. Valoración del estado del Programa de Fluoración de la Sal y sus efectos.

- Promover la implementación de un sistema de vigilancia de la salud bucal en la población: implementar un Sistema Único de Registro Nacional de Salud Bucal.

La atención odontológica, entonces, es incluida en el sistema reconociéndose su importancia en la salud general de las personas. Si bien la financiación para sustentarse a través del Fondo Nacional de Salud (FONASA) se ha planteado sobre la gestión de un sistema solidario y proporcional a las posibilidades de aportes de los ciudadanos, no es suficiente para solventar todos los tratamientos odontológicos. Particularmente la rehabilitación prostodóntica y el tratamiento de maloclusiones no han podido ser cubiertas.

Por otro lado, se ha tratado positivamente la prevención de caries y paradenciopatias. Se ha incursionado en tratamientos restaurativos de poca complejidad sin compromiso del órgano dentino pulpar, se realizan cirugías, tratamiento de patologías tumorales, algunos tratamientos de prótesis removibles, ortopedia interceptiva en niños, atención de malformaciones, diagnóstico imagenológico.

La atención en consultorio privado ha disminuido en general, pero cuando la complejidad de las patologías es mayor, los pacientes recurren a ella, en caso de que puedan acceder económicamente.

Desde el Ministerio de Salud Pública se han implementado algunos programas de salud bucal que priorizan a embarazadas, niños y adolescentes que se presentan a continuación en el próximo apartado. 


\section{Políticas y estrategias aplicadas en el país para mejo- rar la salud bucal}

En 2005 se creó el Programa Nacional de Salud Bucal Escolar para la educación, promoción y asistencia odontológica de escolares de todo el país.

El programa fue impulsado por la Sra. María Auxiliadora Delgado, esposa del presidente de la República Dr. Tabaré Vázquez, y dependía directamente de Presidencia. El objetivo del programa fue promover la salud bucal infantil para un desarrollo integral de la infancia con acciones educativas y preventivas. Se atendió a miles de niños en todo el país, en especial a aquellos de contexto socioeconómico crítico y de zonas rurales, favoreciendo las acciones comunitarias.

Se fundamentó en la promoción de salud y prevención, mostrando a los niños la importancia de su salud bucal, creando hábitos saludables desde edades tempranas al promover el autocuidado, y una alimentación saludable para mejorar las condiciones de vida. La promoción llegó a escuelas privadas por material educativo.

A partir del $1^{\circ}$ de enero de 2019 el Programa de Salud Bucal pasó a depender de la Administración de Salud de los Servicios del Estado (ASSE).

Desde 2017 se ha comenzado a trabajar con la Historia Clínica Electrónica Odontológica, para que la información pueda ser analizada y se mida el impacto del programa por el Ministerio de Salud Pública.

Cuando los niños cuentan con prestadores privados, la asistencia es realizada por dichos centros sanitarios y, en los que no, se los deriva a la red asistencial de ASSE.

El equipo técnico cuenta con 75 Doctores en Odontología y 11 Higienistas en Odontología.

\section{Recursos humanos frente a la situación de la salud bucodental}

Uruguay cuenta con los siguientes recursos humanos en Odontología: "3500 odontólogos activos, 200 higienistas, 600 asistentes dentales titulados y 800 técnicos protésicos. En el sector público trabajan 407 odontólogos y 89 tecnólogos en odontología (higienistas y asistentes) según el documento de trabajo para el taller Plan-Estratégico de la Facultad. Existían organizaciones que agrupaban aproximadamente 900 odontólogos bajo la forma de cooperativa, distribuidos de la siguiente forma: 600 en el interior del país y 300 en Montevideo" (Facultad de Odontología, Universidad de la República. Documento de trabajo).
Con los planes de estudio actualizados desde 2011 para la Carrera de Odontología y para las tres carreras tecnológicas de la FO.UdelaR, se constató en 2018 el ingreso de 260 estudiantes para Doctor en Odontología, 112 para Asistente Dental, 103 para Higienista y 70 para Laboratorista.

Cabe destacar que el volumen del estudiantado activo supera estas cantidades y está compuesto por cerca de 2500 alumnos que han desarrollado alguna actividad curricular en los últimos dos ańos.

Según el último informe anual de la Facultad de Odontología de la UdelaR a diciembre de 2019, el egreso de las cuatro carreras que se imparten es el que se observa en la tabla 2.

En cuanto a los posgrados profesionales, egresan aproximadamente 20 especialistas por año, distribuidos en las disciplinas de: Odontopediatría, Implantología, Periodoncia, Endodoncia, Ortodoncia, Prostodoncia, Cirugía, Gerodontología y Restauradora ${ }^{20}$.

\section{Resultados obtenidos en los últimos 10 años}

En los últimos años se ha podido dar respuesta a diferentes necesidades de la población, pero falta camino por recorrer. En el ámbito del SNIS se atendieron primordialmente embarazadas y niños. Es de esperar que se puedan ir complementando y aumentando las prestaciones y que se llegue a todos los grupos etarios.

Las dos Facultades de Odontología tienen en sus planes de estudio un semestre para prácticas extramurales, que pueden ser hechas en instalaciones públicas de ASSE, policlínicas u hospitales, escuelas, centros de salud zonales de las Intendencias Municipales. La AOU y centros privados de perfeccionamiento y formación disciplinar (Ortodoncia en particular) también atienden pacientes que puedan asumir costos moderados.

Las patologías complejas por caries, paradenciopatías, tumores, ortodoncia, rehabilitación protésica, que no son tratadas a nivel SNIS y que no pueden ser derivadas a atención particular porque los pacientes no tienen solvencia económica, son atendidas aunque en número inferior a la demanda en las facultades de odontología también.

Los desórdenes craneomandibulares, tan prevalentes en los uruguayos, también pueden ser tratados en la Facultad de Odontología UdelaR a nivel de grado y postgrado.

La FO.UdelaR tiene una demanda anual de aproximadamente 5000 pacientes que solicitan atención, pero se

Tabla 2. Egreso de cuatro carreras de Odontología en la Facultad de Odontología de la UdelaR, 2019

\begin{tabular}{lcccc}
\hline Carrera & Varones & Mujeres & Total & Porcentaje \\
\hline Doctor en Odontología & 12 & 58 & 70 & 31,4 \\
Laboratorista en Odontología & 6 & 32 & 38 & 52 \\
Asistente en Odontología & 3 & 49 & 62 & 23,5 \\
Higienista en Odontología & 7 & 55 & 194 & 222 \\
Total egresados & 28 & 100 & 28 \\
\hline
\end{tabular}


puede dar cobertura solamente a la mitad de esas personas (este número de consultas no incluye las urgencias, emergencias ni tratamientos quirúrgicos que alcanzan a 10000 actos odontológicos y son todos atendidos).

Los pacientes con malformaciones congénitas, pérdida de tejidos por neoplasias, traumatismos craneofaciales que han mutilado anatomía y funcionalidad, o defectos en otros territorios no odontológicos como son pérdida de nariz, oreja, dedos, globo ocular, y muchas otras necesidades que requieren rehabilitación protética son atendidos en la FO.UdelaR en el Servicio de Prótesis Buco Maxilo Facial, reconocido a nivel nacional y fuera del país.

La FO.UdelaR ha realizado varios convenios para tratar diferentes colectivos y se destacan dos de ellos por la numerosidad de personas que se atienden. Hace veinte años existe un convenio de atención integral a funcionarios no docentes de la UdelaR (5 000 funcionarios en actividad aproximadamente). Otro de ellos es el Programa de Salud Bucal del Plan de Integración Socio Habitacional Juntos del Estado (que abarca unos 15000 hogares) y realiza en la FO.UdelaR la atención de los pacientes odontológicos desde 2012, brindando asistencia integral con carácter multidisciplinario a más de 400 personas por año en la propia facultad. Además, en forma descentralizada y ambulatoria, utiliza tres vehículos, dos de ellos equipados con unidades odontológicas y uno para traslado de profesionales para trabajar en todo el país ${ }^{21}$.

En estos años, ha habido otras iniciativas para atender problemáticas de diferentes comunidades. Una de ellas fue en 2012 la que surgió de un llamado concursable de la Junta Nacional de Drogas que buscaba solucionar diferentes problemáticas derivadas de la drogadicción. La Junta apoyó con financiación uno de los proyectos presentado por un equipo interdisciplinario encabezado por un odontólogo, el Dr. Rotemberg, en el que además de investigar las patologías orales de los pacientes en tratamiento por consumo de drogas, brindaba tratamiento restaurativo y rehabilitador a quienes estaban reintegrándose a su vida en sociedad ${ }^{8}$.

\section{Conclusiones}

Las patologías más prevalentes en la población uruguaya son la caries dental y las paradenciopatias. La problemática de la salud bucal de la población uruguaya, se aborda desde los distintos escenarios sanitarios y educativos; los que brindan una permanente estrategia preventiva dando respuesta a las necesidades terapéuticas, en lo posible.

Si bien la financiación para sustentar las necesidades odontológicas se sostiene por un sistema solidario y proporcional a las posibilidades de aportes de los ciudadanos, este no es suficiente para solventar todos los tratamientos odontológicos. La rehabilitación y el tratamiento de maloclusiones no son cubiertos.

La academia juega un rol fundamental en el manejo de la situación. Es su deber como institución encargada de buscar y difundir el saber científico, ponerlo a disposición de la sociedad dando todo su caudal humano para que las personas puedan lograr el equilibrio bio-psico-social. He ahí el sentido circular al que se hacía referencia al inicio de este manuscrito.

La academia busca el conocimiento; da herramientas para responder a las necesidades y a los problemas que recibe de la comunidad cuando sale a buscar dicho conocimiento. He ahí el sentido ético de pensar, sentir y trabajar por la salud de las personas.

\section{Referencias bibliográficas}

1. Instituto Nacional de Estadística. Anuario Estadístico 2019. 96a versión. República Oriental del Uruguay. [Citado 5 abril 2020]. Disponible en:http://www.ine. gub.uy/documents/10181/623270/Anuario+Estadistico+2019/f854fb27-ad7f-4ce3-8c37-005ade0a6140

2. Ministerio de Salud Pública. Ley No18.211 de Creación del Sistema Nacional Integrado de Salud. [Citado 5 abril 2020]. Disponible en: http://www.mysu.org.uy/wp-content/uploads/2014/11/Ley-18211-sistema-nacional-integrado-de-salud_-creacion.pdf

3. Ministerio de Salud Pública. Programa Nacional de Salud Bucal. [Citado 2 abril 2020]. Disponible en: https:// www.gub.uy/ministerio-salud-publica/politicas-y-gestion/programas/programa-nacional-de-salud-bucal

4. Lorenzo-Erro S, Álvarez R. Relevamiento nacional de salud bucal de jóvenes y adultos uruguayos 2011. Universidad República de Uruguay Facultad de Odontología. Servicio de Epidemiología y Estadística, Cátedra de Odontología Social.Uruguay,2018. [Citado 5 abril 2020]. Disponible en: https://www.colibri.udelar.edu. uy/jspui/bitstream/20.500.12008/18508/1/Lorenzo_ Encuesta.pdf.

5. Riva R, Sanguinetti M, Rodríguez A, Guzzetti L, Lorenzo S, Álvarez R; et al. Prevalencia de trastornos témporo mandibulares y bruxismo en Uruguay: PARTE I. Odontoestomatología [Internet]. 2011 Mayo [citado 03 Mayo 2020] ;13(17):54-71. Disponible en: http:// www.scielo.edu.uy/scielo.php?script $=$ sci_arttext\&pi$\mathrm{d}=$ S1688-93392011000100007\&lng=es.

6. Angulo M, Bianco P, Cuitiño E, Silveira A. Relevamiento y análisis de caries dental, fluorosis y gingivitis en adolescentes escolarizados de 12 años de edad en la ROU. Primer reporte Nacional. Montevideo, 2010 - 2011. [citado 04 de Mayo 2020]. Disponible en : https://www. colibri.udelar.edu.uy , jspui

7. Techera A, Villamonte 1, Pardo L, López Jordi M. Comparación de la situación de salud bucal en nińos uruguayos de 8 a 12 ańos diabéticos y no diabéticos. Odontoestomatología [Internet]. 2018 Dic [citado 26 abril 2020] ; 20(32):84-91. Disponible en: http:// www.scielo.edu.uy/scielo.php?script=sci_arttext\&pi$\mathrm{d}=$ S1688-93392018000200084\&lng=es. http://dx.doi. org/10.22592/ode2018n32a11.

8. Rotemberg E, Salveraglio I, Kreiner M, Piovesan S, Smaisik K, Ormaechea R, et al. Estado dental y periodontal de población en tratamiento por consumo de drogas: Estudio piloto. Odontoestomatología [Internet]. 2015 Mayo [citado 04 de Mayo 2020]; 17(25):34-39. Disponible en: http://www.scielo.edu.uy/scielo.php?script=sci_arttext\&pid=S1688-93392015000100005\&lng=es. 
9. Olmos P, Piovesan S, Musto MS, Lorenzo S, Alvarez R. La caries dental, enfermedad oral mas prevalente. Primer Estudio poblacional en jóvenes y adultos uruguayos del interior del país. Odontoestomatología. 2013;XV:26-34.

10. Lorenzo S, Piccardo V, Alvarez F, Massa F, Alvarez-Vaz R. Enfermedad Periodontal en la población joven y adulta uruguaya del interior del país. Un estudio poblacional. Odontoestomatología. 2013;XV:35-46.

11. Ourens M, Roger KC, Hilgert J, Lorenzo S, Neves F, Alvarez-Vaz R,Abbeg C. Prevalencia de maloclusiones en adolescentes y adultos jóvenes del interior del Uruguay en el relevamiento nacional de salud bucal 2010-2012. Odontoestomatología. 2013;XV:47-57.

12. Laguzzi PN, Schuch HS, Medina LD, et al. Tooth loss and associated factors in elders: results from a national survey in Uruguay. J Public Health Dent. 2016 Mar;76(2):143-151. DOI: 10.1111/jphd.12123.

13. Casnati B, Álvarez R, Massa F, Lorenzo S, Angulo M, Carzoglio J. Prevalencia y factores de riesgo de las lesiones de la mucosa oral en la población urbana del Uruguay. Odontoestomatología. [Internet]. 2013 Jun [citado 04 Mayo 2020] ;15(spe):58-67. Disponible en: http://www.scielo.edu.uy/scielo.php?script=sci_arttext\&pid=S1688-93392013000200007\&lng=es.

14. Lorenzo, S., Alvarez, R. Prevalencia de caries en escolares de 12 años de diferente nivel socioeconómico, Montevideo, Uruguay, 2003. Odontoestomatología. 2009;11(13):27-36.

15. López Jordi Mdel C, Cortese SG, Álvarez L, Salveraglio I, Ortolani AM, Biondi AM. Comparison of the prevalence of molar incisor hypomineralization among children with different health care coverage in the cities of Buenos Aires (Argentina) and Montevideo (Uruguay)].
Salud Colect. 2014 Aug;10(2):243-51. DOI: 10.1590/ S1851-82652014000200008.

16. Alvarez Loureiro L, Fabruccini Fager A, Alves LS, Alvarez Vaz R, Maltz M. Erosive tooth wear among 12-year-old schoolchildren: a population-based cross-sectional study in Montevideo, Uruguay. Caries Res. 2015;49(3):21625. DOI: $10.1159 / 000368421$

17. Caviglia I. Desgaste erosivo en preescolares de 5 ańos de Montevideo - Uruguay: estudio trasversal [en línea] [Tesis de maestría]. [Montevideo]: Facultad de Odontología UdelaR. 2019. 56p.

18. Primer Observatorio de Investigación de Caries Dental en América Latina [citado 04 Abril 2020] Disponible en: https:/odon.edu.uy/sitios/odontopediatria/investigacion

19. Rotemberg E, Francia A, Muñoz M. Relevamiento de caries en población adolescente y adulta joven afro-uruguaya. Salud Militar. 2017;36(2):25-30.

20. UdelaR. Facultad de Odontología. Síntesis Estadística de la Universidad. Sistema de gestión de Bedelías (SGB). Montevideo. 2019 [citado 04 Abril 2020] Disponible en: .http://gestion.udelar.edu.uy/planeamiento/

21. UdelaR. Facultad de Odontología. Montevideo 2020. Plan Juntos Odontología. [citado 04 Abril 2020] . Disponible en: https://odon.edu.uy/sitios/planjuntos/ 